\title{
DIATERMIA POR ONDAS CURTAS: ANÁLISE DA TEMPERATURA CORPORAL SUPERFICIAL POR TERMOGRAFIA
}

\section{Shortwave diathermy: surface analysis of body temperature in infrared}

\author{
Sergio Fernando Zavarize \\ Doutor em Psicologia pela PUC-Campinas, Brasil. \\ sergio@fisiozavarize.com.br
}

\section{Anderson Martelli}

Especialista em Patologia Clínica pela Faculdade de Ciências Médicas - UNICAMP Campinas - SP martellibio@hotmail.com

\section{Samara Aparecida Machado}

Fisioterapeuta graduada pela Pontifícia Universidade Católica de Campinas. Especialista em Pilates. samarafisioterapia@gmail.com

\section{Estela Maria Correia Sant'Ana}

Doutora em Ciências Fisiológicas UFSCar, Brasil..

estela@ibramed.com.br

\section{Resumo}

Introdução: Poucos estudos relacionam os efeitos da diatermia por ondas curtas (DOC) e a área de aquecimento promovido por sua aplicação. Objetivo: O objetivo do trabalho foi avaliar o comportamento da temperatura superficial corporal antes e após a aplicação de DOC por meio da termografia cutânea. Materiais e métodos: A análise da temperatura corporal no local da aplicação foi realizada através da captura da imagem por meio de câmera termográfica após quinze minutos de repouso do paciente em sala com temperatura controlada e no segundo momento, a análise termográfica após a aplicação de DOC, realizado pelo equipamento Thermopulse Ibramed, durante 20 minutos. As áreas de aplicação foram a coluna lombar, ombro e joelho. Resultados: Os resultados demonstraram uma melhora significativa na dor entre as temperaturas iniciais e após a aplicação da DOC, o que indica a eficiência do equipamento utilizado como emissor de calor na intensidade desejada. Conclusão: $O$ aumento da temperatura local através da DOC favoreceu a recuperação de lesões musculoesqueléticas pós-agudas, rigidez após longos períodos de imobilização e redução da intensidade da dor nos processos inflamatórios.

Palavras-Chave: Diatermia, Ondas curtas, Temperatura, Termografia, Dor. 


\section{Abstract}

Introduction:Few studies are related to the effects of short-wave diathermy (SWD) and the heating area promoted by its application. Objective: The objective of this research is to evaluate the behavior of the body temperature before and after the application of SWD through the skin thermography. Materials and methods: The analysis of body temperature at the site of application was done by capturing the image by a thermographic camera after fifteen minutes to rest of patient at room with controlled temperature and later, was made the thermographic analysis after applying SWD performed by the equipment Thermopulse Ibramed for 20 minutes. The areas chosen for evaluation are the thoracic spine, lumbar spine, shoulder and knee. Results: The results showed a significant improvement in pain between the initial temperatures and after implementation of the DOC, which indicates the efficiency of the equipment used as heat emitter on the desired intensity. Conclusion: The increase in local temperature by DOC led to the recovery of post-acute musculoskeletal injuries, stiffness after long periods of immobilization and reduction in intensity of the pain in inflammatory processes.

Keywords: Diathermy, Shortwave Temperature, Thermography, Pain.

\section{INTRODUÇÃO}

As temperaturas no corpo humano, quando normais, podem ser divididas em temperatura central e temperatura cutânea. A temperatura central, ou seja, dos tecidos profundos do corpo, permanece quase que exatamente constante, com variação de mais ou menos $0,6^{\circ} \mathrm{C}$, exceto quando o indivíduo contrai uma doença onde se manifeste um estado febril. Já a temperatura cutânea, aumenta e diminui de acordo com a temperatura do ambiente.

Ela é importante no que se refere à capacidade da pele de não perder calor para o meio externo. Em geral, a temperatura corporal média situa-se entre $36,7^{\circ} \mathrm{C}$ até mais de $37^{\circ} \mathrm{C}$, quando a medida é efetuada na boca (GUYTON \& HALL, 2011; BUGGY \& CROSSLEY, 2000).

A manutenção da temperatura dentro dos padrões normais nos animais homeotermos, assim como o homem, é uma função muito importante do sistema nervoso autônomo. Já que com pequenas alterações da temperatura central, podem ocorrer alterações metabólicas e enzimáticas importantes (BUGGY \& CROSSLEY, 2000; BRAZ, 2005; WILMORE et al., 2010).

A regulação da temperatura do corpo é realizada por um sistema de controle fisiológico, que consiste em termorreceptores centrais e periféricos, um sistema de condução aferente, o controle central de integração dos impulsos térmicos (GUYTON \& HALL, 2011), uma região do cérebro denominado núcleo pré-óptico do hipotálamo anterior (WILMORE et al., 2010) e um sistema de respostas eferentes levando a respostas compensatórias (GUYTON \& HALL, 2011).

Portanto, em relação às reações físiológicas ao calor, o organismo reage localmente, aumentando a liberação de acetilcolina. Esta, por sua vez, dilata os vasos sanguíneos e facilita o 
fluxo de sangue. O fluxo sanguíneo aumentado representa uma reação orgânica termolítica e, neste local, favorece a eliminação de subprodutos indesejáveis do metabolismo (catabólitos) que são, muitas vezes, responsáveis por dores e desconfortos musculares. Além dessas ações, a vasodilatação também favorece a migração dos macrófagos, contribuindo, assim, para o combate contra os processos infecciosos (BRAZ, 2005).

A termoterapia pode ser definida como uma forma de terapia que se utiliza dos efeitos da temperatura, isto é, do calor (adição) e do frio (subtração) sobre os tecidos do corpo (BRUNO et al., 2001). Por sua vez, a Diatermia consiste no uso de um transmissor de ondas curtas com o objetivo de provocar o aquecimento de tecidos internos do corpo (BRAZ, 2005; BRASILEIRO et al., 2007).

As correntes elétricas de alta frequência têm sido utilizadas na área biomédica desde 1890. Com o desenvolvimento de novas técnicas surgiu a diatermia que utiliza a produção de calor terapêutico proveniente das correntes de alta frequência. As ondas curtas são um recurso de diatermia utilizado pelos fisioterapeutas que converte a energia eletromagnética de alta frequência em energia térmica nos tecidos (SILVA et al., 2007).

A radiação eletromagnética emitida por este recurso é do tipo não ionizante que envia calor para os tecidos mais profundos (SILVA et al., 2007) e apresenta como efeitos terapêuticos a ação antiinflamatória, analgésica, cicatricial, descontraturante e hiperemiante (TEIXEIRA et al., 2001).

Assim sendo, a diatermia por ondas curtas (DOC) é uma modalidade terapêutica que utiliza bandas de radiofrequência geralmente centralizadas em 13,56 MHz, 27,12 $\mathrm{MHz}$ e 40,68 MHz, sendo a frequência mais comumente utilizada de $27,12 \mathrm{MHz}$ com comprimento de onda correspondente de 11,6m (CAMERON, 2009; SHIELDS et al., 2002; HILL et al., 2002) que apesar de rotineiramente empregada entre fisioterapeutas, ainda restam muitas questões relativas à sua aplicabilidade (KITCHEN \& BAZIN, 2003; ROBERTSON et al., 2006).

Este recurso terapêutico trabalha sobre o princípio de que a energia é transferida dentro das camadas de tecidos profundos por uma corrente de alta freqüência e a geração de calor tecidual. Ocorre, portanto, uma agitação das moléculas em resposta a um campo elétrico, convertendo a energia cinética em calor (KITCHEN \& BAZIN, 2003; LONGO \& FIURINI, 2000).

Silva et al. (2007) relatam alguns riscos potenciais que devem ser considerados no uso deste recurso termoterapêutico, como o útero gravídico, podendo levar à má formação fetal, marca-passo cardíaco, com alteração do seu funcionamento, neoplasias e o desenvolvimento de metástase, sangramento ativo (menstruação) com aumento do fluxo sanguíneo e hemorragia, proximidade com outros aparelhos eletrônicos interferindo em seu funcionamento.

Dentre as técnicas de aplicação da DOC, o modo indutivo proporciona um aumento de temperatura nos tecidos profundos predominantemente, já o modo capacitativo ocorre 
principalmente nos tecidos superficiais (KITCHEN \& BAZIN, 2003; ROBERTSON et al., 2009; LONGO \& FIURINI, 2000; PRENTICE, 2002).

A temperatura dos tecidos corporais é em alguns estudos, medida que varia devido à dose, o método e a região mensurada (KITCHEN \& BAZIN, 2003). Nos trabalhos de Oosterveld (1992) que utilizaram o método capacitativo, observou-se um aumento da temperatura da pele em $2,4^{\circ} \mathrm{C}$ e $2,24^{\circ} \mathrm{C}$ respectivamente.

No entanto, as doses ministradas foram diferentes, sendo a primeira utilizada como calor perceptível e a segunda, como calor suportável (ABID et al., 2010). Algumas pesquisas utilizaram outros métodos de aplicação da DOC e também avaliaram a quantidade de calor absorvida pelos tecidos (KITCHEN \& BAZIN, 2003; MURRAY \&KITCHEN, 2000).

A avaliação e medida da temperatura superficial dos tecidos podem ser realizadas por meio de câmeras termossensíveis que se baseia na detecção de radiação térmica emitida - termografia (BALBINOT \& VIEIRA, 2005; MOURA et al., 2011).

A termografia é a técnica que estende a visão humana através do espectro infravermelho. $\mathrm{O}$ infravermelho é uma frequência eletromagnética naturalmente emitida por qualquer corpo, com intensidade proporcional à sua temperatura. São, portanto, emissões de infravermelho através de uma tela, produzindo imagens técnicas chamadas de termogramas, que, em resumo, permite a visualização da distribuição de calor na região focalizada como mostra a figura 1 (OLIVEIRA JR, 2010).
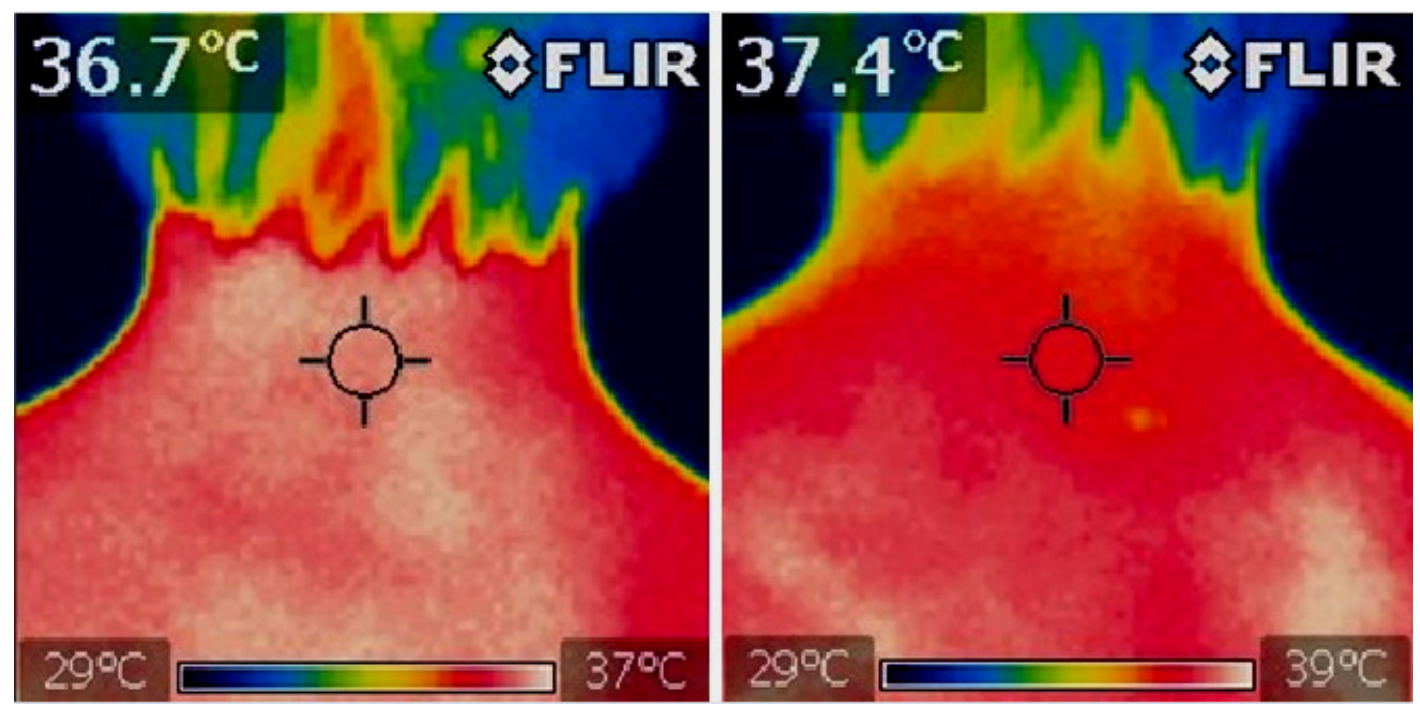

Figura 1. Termogramas da coluna cervical mostrando as variações no calor radiante (infravermelho) que deixam à superfície corpórea antes e após a aplicação da diatermia por ondas curtas.

Este tipo de técnica, embora seja ainda pouco explorado na prática fisioterapêutica é de $\underline{\text { grande importância, pois a distribuição da temperatura pela área de tratamento pode ser relacionada }}$ 


\section{PERSPECTIVAS online}

à eficiência do equipamento em produzir calor na intensidade desejada e em contrapartida, temperaturas muito elevadas podem causar desnaturação proteica com consequentes lesões teciduais (KITCHEN \& BAZIN, 2003; LONGO \& FIURINI, 2000).

Estas lesões são totalmente contrárias aos efeitos esperados pelo uso do calor como terapia. Além disso, este tipo de avaliação pode contribuir para um maior conhecimento dos efeitos e reações do corpo ao calor (KITCHEN \& BAZIN, 2003; LONGO \& FIURINI, 2000).

Assim, o objetivo deste trabalho foi avaliar a temperatura superficial corporal por meio da termografia antes e após aplicação da DOC na região da coluna lombar, ombro e joelho e verificar os efeitos desta técnica sobre a dor mediante avaliação pela Escala Visual Analógica (EVA). As variações das temperaturas foram analisadas nas áreas de aplicação do equipamento Thermopulse Ibramed por meio do software FLIR QuikReport versão 1.2 SP2.

\section{MATERIAIS E MÉTODOS}

Trata-se de uma pesquisa experimental, onde o projeto de estudo foi submetido e aprovado pelo Comitê de Ética em pesquisa da Faculdade de Jaguariúna - FAJ, parecer $n^{\circ}$ 008/2011. O estudo foi realizado em uma clínica de Fisioterapia situada na cidade de Mogi Mirim SP, mediante declaração de autorização. Para a composição da amostra, foram escolhidos de forma aleatória 17 pacientes adultos com idade entre 17 e 83 anos em tratamento de quadros álgicos no ombro, coluna lombar e joelho simultaneamente, sendo aferidos o Peso e a Altura para a realização do Cálculo do índice de massa corporal (IMC). O peso foi aferido em balança digital com capacidade máxima de $150 \mathrm{~kg}$ e divisão de $100 \mathrm{~g}$ e a estatura foi aferida com fita métrica plástica (Sanny Medical) aderida a uma parede sem rodapé, com extensão de 2,00 m, dividida em centímetro e subdividida em milímetros, com visor de plástico e esquadro acoplado a uma das extremidades.

O IMC foi calculado com as medidas de peso e altura, de acordo com a seguinte fórmula IMC $=$ peso $(\mathrm{kg}) /$ altura $^{2}(\mathrm{~cm})$. Os pontos de corte de IMC adotados foram os preconizados pela WHO9, ou seja, baixo peso (IMC < 18,5); eutrofia (IMC 18,5-24,99); sobrepeso (IMC 25-29,99) e obesidade (IMC $\geq 30,00$ ) (FERREIRA et al., 2006).

Os participantes passaram inicialmente por uma triagem considerando os dados de antecedentes pessoais, colhidos a partir do prontuário da anamnese do paciente, sendo considerado como critérios de inclusão o conjunto de queixas álgicas simultâneas no ombro, coluna lombar e joelho, aceite do paciente em participar da pesquisa e assinatura do Termo de Consentimento Livre e Esclarecido. 
Como critérios de exclusão foram adotados: queixas álgicas em apenas um dos lugares retratados na pesquisa, ferimentos de pele em uma ou mais regiões a serem tratadas e os quadros agudos de processos degenerativos avançados onde o tratamento com calor profundo geralmente não é indicado conforme retratado por De Assunção et al. (2012), uma vez que a elevação da temperatura intra-articular pode levar ao aumento da atividade enzimática responsável pela degeneração articular.

Previamente à realização dos protocolos experimentais, os participantes da pesquisa permaneceram em repouso por 15 minutos para ajuste da temperatura corporal em uma sala com temperatura aproximada de $28,7 \pm 2{ }^{\circ} \mathrm{C}$. A mensuração da temperatura externa foi aferida por meio do equipamento FLIR.

Em seguida foi realizada a captura da imagem através de uma câmera infravermelha marca FLIR modelo i5 antes da aplicação da DOC no local a ser tratado de acordo com a queixa e/ou patologia do paciente, preconizando neste estudo as respectivas regiões: coluna lombar, ombro e joelho.

Posteriormente, foi realizada a aplicação de DOC com o equipamento Thermopulse Ibramed (Indústria Brasileira de Equipamentos Médicos EIRELI, Amparo, Brasil), na forma contínua, por um tempo de 15 minutos com emissão de calor em níveis suportáveis e de forma agradável para o paciente, sendo realizada a captação das imagens locais com a câmera termográfica imediatamente após o tratamento.

Com o intuito de averiguar a eficácia do equipamento utilizado, foi realizada a avaliação da dor pela Escala Visual analógica (EVA), imediatamente antes e após a realização das sessões. A Escala Visual Analógica é um instrumento amplamente utilizado, que tem a função de avaliar a percepção da intensidade da dor. É constituída por uma linha reta cujo extremo esquerdo aponta para a "ausência de dor" e o extremo direito para a "pior dor possível".

Os valores desta escala variam de zero a dez e o paciente é instruído a marcar o ponto que indique a intensidade da sua dor. Escalas de itens simples, como esta, são válidas e extremamente sensíveis para mudanças no estado do paciente (BRYCE et al., 2007), sendo um instrumento importante para verificar a percepção de incômodo da dor e para analisar se a intervenção direcionada à redução da dor está sendo efetiva.

Quanto ao posicionamento das placas da DOC e a aferição da temperatura, este estudo preconizou a metodologia utilizada por Robertson et al. (2009). Para os tratamentos de ombro foram realizadas de forma capacitiva, com os eletrodos posicionados de forma longitudinal, os pacientes permaneceram sentados tanto para aplicação quanto para o registro das imagens e as fotografias foram tiradas a uma distancia de $80 \mathrm{~cm}$. 
Os tratamentos de coluna lombar foram realizados com os eletrodos capacitivos posicionados de forma coplanares com o paciente em decúbito dorsal, as imagens foram capturadas imediatamente após os tratamentos com os pacientes de pé a uma distância de $130 \mathrm{~cm}$ e os tratamentos da área de joelho foram realizadas com o eletrodo indutivo posicionados na região lateral (externa) do joelho, com o membro estendido e as imagens capturadas a uma distancia de 70 $\mathrm{cm}$ com o paciente sentado e joelho fletido a um anglo de $90^{\circ}$.

As imagens obtidas antes e após a aplicação da DOC foram analisadas por meio do software FLIR QuikReport versão 1.2 SP2 para a mensuração da temperatura corporal. Ao final da coleta de dados, os resultados foram analisados estatisticamente com o programa Statistical Package for the Science (SPSS), versão 18 para Windows.

As variáveis contínuas foram expressas em médias e desvios-padrão Os dados referentes às temperaturas aferidas foram tratados meio do teste $t$ de Student quando $\mathrm{p} \leq 0,05$. Os resultados da escala visual analógica (EVA) para dor foram analisados pela correlação de Pearson com o mesmo índice de significância. A finalização deste trabalho ocorreu com a comparação dos dados observados com os resultados descritos na literatura.

\section{RESULTADOS E DISCUSSÃO}

Em relação à distribuição por sexo, dos 17 pacientes selecionados aleatoriamente na clínica de fisioterapia, houve predominância do sexo feminino com 10 pacientes, respondendo por 58,8\%, para 7 pacientes do sexo masculino $(41,2 \%)$. Quanto à idade, os voluntários participantes do estudo apresentavam-se na faixa etária variando entre 17 e 83 anos, uma média de idade geral de 50,7 anos sendo apresentada uma média de idade feminina de 49,6 anos e masculina de 52,1 anos.

$\mathrm{Na}$ análise da amostra quanto ao peso, altura e IMC, a média geral foi de 72,7 kg, 1,66 m e 26,26 respectivamente, sendo a média feminina de 69,9 kg, 1,60 m e 27,21, e masculina de 76,6 kg, $1,75 \mathrm{~m}$ e 24,9, concomitantemente como mostra a Tabela 1.

Tabela 1. Distribuição das médias de peso, altura e índice de massa corporal (IMC) entre os sexos.

\begin{tabular}{lccc}
\hline Sexo & Peso em kg & Altura em metros & IMC \\
\hline Feminino & 69,9 & 1,60 & 27,21 \\
Masculino & 76,6 & 1,75 & 24,90 \\
Média & 72,7 & 1,66 & 26,26 \\
\hline
\end{tabular}

IMC: baixo peso (IMC < 18,5); eutrofia (IMC 18,5-24,99); sobrepeso (IMC 25-29,99) e obesidade (IMC $\geq 30,00$ ). 
A Tabela 1 apresenta os IMC dos participantes. O IMC, atualmente utilizado foi proposto em 1835, pelo estatístico belga Lambert Adolphe Jacques Quételet, e adotado em 1997 pela Organização Mundial da Saúde (OMS), como referência de medida para a obesidade.

No respectivo estudo este índice é importante e interfere nas taxas de calor observadas, sendo que quanto maior o IMC do paciente, maior foi a variação da temperatura local antes e após a aplicação de DOC, corroborando com dados existentes na literatura especializada onde aponta, que quanto maior a porcentagem de gordura e IMC, menor seria a perda da temperatura.

O tecido adiposo por constituir depósitos localizados em diversas regiões do organismo envolvendo-o, ou mesmo se infiltrando em órgãos e estruturas internas incluindo a derme e tecido subcutâneo, é considerado um excelente isolante térmico com um papel importante na manutenção da temperatura corporal.

Segundo Low e Reed (2001), o tecido adiposo absorve mais energia térmica e se aquece mais rápido que o tecido muscular, sendo este calor conduzido para os tecidos mais profundos e dificultando sua dissipação para o ambiente devido sua baixa condução de calor e seu potencial isolante, fatores que favoreceram os resultados obtidos neste estudo.

Neste estudo, na avaliação da temperatura superficial corporal antes e após aplicação da DOC na região do ombro, coluna lombar e joelho por meio da termografia com o aparelho Thermopulse Ibramed as variações das temperaturas foram consideradas extremamente significante conforme demostrado na tabela 2.

Tabela 2. Temperaturas mínima, máxima, média e desvio padrão da média da temperatura tecidual superficial, aferidas por termógrafo das áreas antes do tratamento e depois de tratadas com DOC.

\begin{tabular}{|c|c|c|c|c|c|}
\hline Área tratada & T Mínima & T Máxima & T Média \pm DP & $\mathbf{p} \leq$ & \\
\hline Ombro antes & 29,3 & 37,1 & $34,4 \pm 2,0$ & & \\
\hline Ombro depois & 32,5 & 38,9 & $36,4 \pm 1,8$ & 0,001 & $6,0^{\circ} \mathrm{C}$ \\
\hline Joelho antes & 30,8 & 36,1 & $33,9 \pm 1,4$ & & \\
\hline Joelho depois & 32,0 & 40,6 & $37,1 \pm 2,4$ & 0,001 & $4,9^{\circ} \mathrm{C}$ \\
\hline Lombar antes & 28,4 & 36,5 & $34,0 \pm 2,1$ & & \\
\hline Lombar depois & 35,4 & 40,8 & $38,5 \pm 1,5$ & 0,001 & $7,6^{\circ} \mathrm{C}$ \\
\hline
\end{tabular}

$\mathrm{T}$ mínima: menor temperatura obtida; T máxima: maior temperatura obtida; T média: temperatura média na amostra $(\mathrm{p} \leq 0,05=$ significativo; $\mathrm{p} \leq 0,01=$ muito significativo. $\mathrm{p} \leq 0,001=$ extremamente significativo $)$. 
Os resultados apresentaram os valores de 6,0 para ombro, 4,9 para joelho e 7,6 para aplicação na coluna lombar. Embora se esperasse à princípio atingir um nível de significância mínimo de $5 \%$, isto é, com um p $\leq 0,05$ entre as correlações a serem observadas, o teste $t$ mostrou uma diferença significativa entre as temperaturas antes e após a aplicação de DOC com $\mathrm{p} \leq 0,001$.

Na região do ombro, foi possível observar uma variação mínima positiva de temperatura de $0,2^{\circ} \mathrm{C}$ e uma variação máxima de $3,8^{\circ} \mathrm{C}$. Na região do joelho, observou-se uma variação mínima positiva de $0,4^{\circ} \mathrm{C}$ e máxima de $8,6^{\circ} \mathrm{C}$ e na ultima região analisada - coluna lombar, a variação mínima positiva ficou em $1^{\circ} \mathrm{C}$ e a máxima em $9^{\circ} \mathrm{C}$ apresentando uma média de temperatura expressiva após a aplicação de DOC como pode ser observado no gráfico 1.

\section{Gráfico das médias das Temperaturas antes e depois das aplicações de Ondas Curtas}

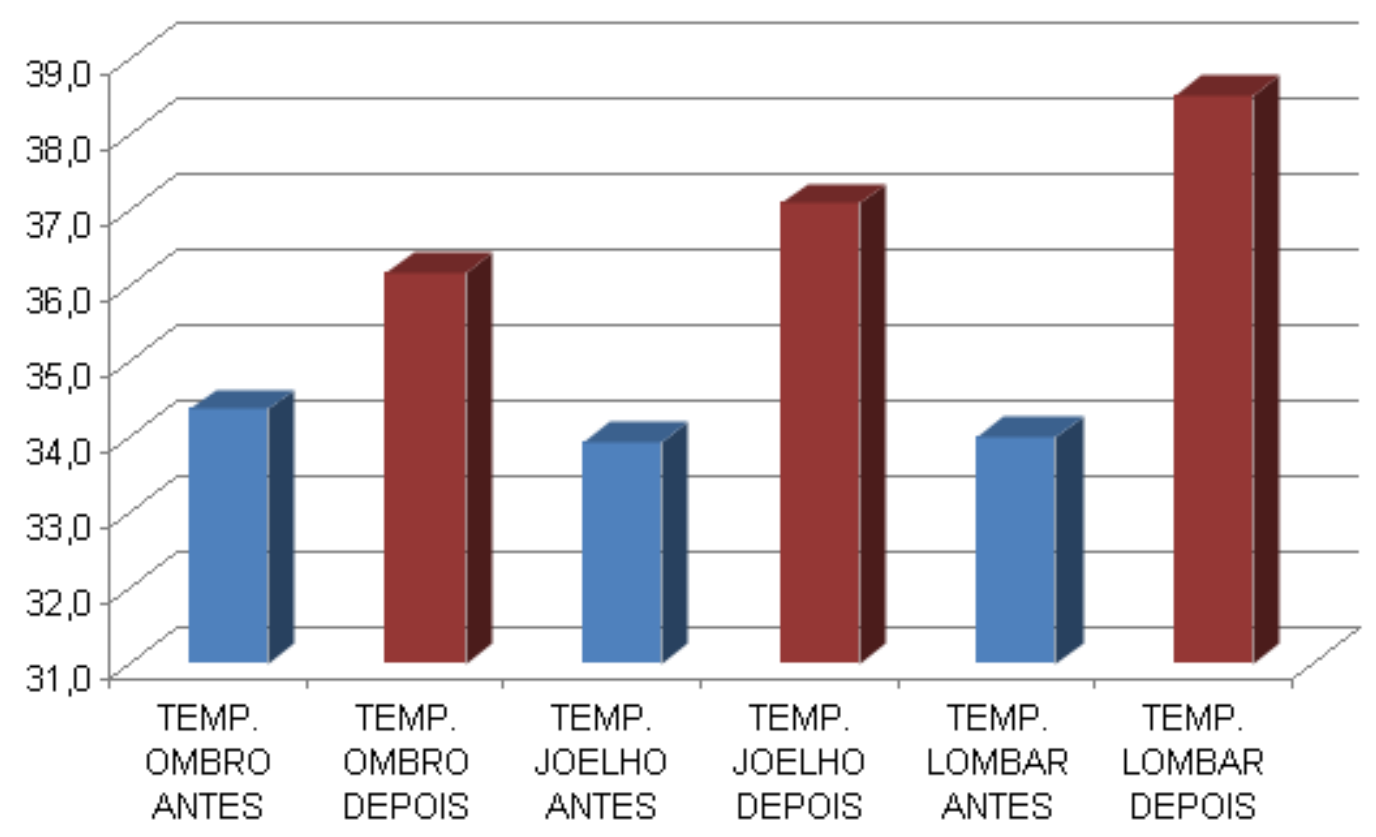

Gráfico 1. Médias das Temperaturas antes e depois das aplicações da DOC.

As variações de temperatura observadas neste estudo favorecem primariamente os reflexos dos termorreceptores quando se trata de estruturas subcutâneas. Assim, a estimulação dos receptores sensoriais de calor pode ativar o mecanismo da comporta na modulação da dor, fenômeno este que não está completamente elucidado, sendo descrito na literatura especializada uma redução dos estímulos aferentes primários nociceptivos em nível tecidual, decréscimo da ativação dos elementos do sistema nervoso periférico (SNP) e sistema nervoso central (SNC) e consequentemente o alivio de quadros dolorosos. 
Secundariamente, o aquecimento de regiões corporais aumenta a taxa metabólica local conduzindo ao aumento da pressão hidrostática intravascular, vasodilatação arteriolar e o aumento de fluxo sanguíneo dos capilares, o que aumenta o fornecimento de oxigênio, anticorpos, leucócitos, citocinas e outros nutrientes e enzimas que aceleram a resolução de processos inflamatórios.

A indução local do aumento da temperatura aumenta também a elasticidade com a diminuição da viscosidade do tecido conjuntivo, sendo esta uma consideração importante nas lesões articulares pós-aguda ou depois de longos períodos de imobilização resultando na redução da intensidade da dor.

Os resultados em relação à avaliação da dor mostraram uma média de 6,9 pela escala EVA antes do início da primeira aplicação e 5,7 após a segunda aplicação. Ao final do tratamento de dez sessões, obteve-se o valor médio para a dor de 2,3 na escala EVA. Esses resultados foram significativos apresentando uma melhora em média de 18,4\% da primeira para a segunda aplicação e de $67,2 \%$ para o tratamento completo de dez sessões.

A correlação de Pearson apresentou um nível de significância de $\mathrm{p} \geq 0,01$ entre os todos os valores encontrados. Esses valores indicam a eficácia do equipamento utilizado e consequentemente, confirmam os efeitos positivos do tratamento que utiliza a Diatermia por Ondas Curtas.

\section{CONCLUSÃO}

O tratamento por DOC é uma modalidade de tratamento conhecida há mais de 50 anos sendo utilizada para o tratamento de uma ampla gama de patologias e no gerenciamento de condições não-ortopédicas que também requerem estes benefícios, sendo uma modalidade segura, confortável e efetiva no tratamento de processos inflamatórios.

Com a aplicação da DOC, a temperatura no local de aplicação apresentou uma elevação como verificado pela termografia e os pacientes tratados por essa técnica apresentaram diminuição dos quadros dolorosos aferidos pela escala EVA.

Raras pesquisas relatam os efeitos térmicos e fisiológicos da emissão de DOC em relação à área de aquecimento promovido por sua aplicação. Por esta razão é de grande importância e relevância a realização de estudos desse tipo, pois as informações colhidas são fundamentais para uma avaliação e aprimoramento de novos protocolos, favorecendo um direcionamento da produção de calor no local da lesão, produtos e tecnologias visando o bem estar e a melhoria da qualidade de vida dos pacientes que fazem uso da DOC no tratamento de quadros álgicos. 


\section{REFERÊNCIAS BIBLIOGRÁFICAS}

ABIB R. T.; MARQUES F. O.; CASALI E. A.; PINHO A. S.; ZARO M. Avaliação da diatermia por ondas curtas contínuo na temperatura superficial do músculo quadríceps. Revista Ciência e Movimento, v. 23: p. 69-77, 2010.

BALBINOT L. F.; VIEIRA L. R. Avaliação objetiva da síndrome dolorosa miofascial: uso da termografia antes e após tratamento associando mesoterapia a bloqueio anestésico. Acta Fisiátrica, v. 12, n. 3: p. 115-117, 2005.

BRASILEIRO, J. S.; FARIA, A. F.; QUEIROZ, L. L. Influência do resfriamento e do aquecimento local na flexibilidade dos músculos isquiotibiais. Revista Brasileira de fisioterapia, v. 11, n. 1: p. 57-61, 2007.

BRAZ, J. R. C. Fisiologia da termorregulação norma. Anais do V Simpósio Brasileiro de Hipertermia Maligna. Revista Neurociências, v. 13, n. 3: p. 13-17, 2005.

BRUNO, A. A. et al.. Meios físicos em reabilitação. In: LIANZA, Sérgio. Medicina de Reabilitação. 3. ed. Rio de Janeiro: Guanabara Koogan, 2001.

BRYCE, T. N, et al. Pain After Spinal Cord Injury: An Evidence-based Review for Clinical Practice and Research. J. Spinal Cord. Med, v. 30, n. 5: p. 421-40, 2007.

BUGGY D. J; CROSSLEY A. W. A. Thermoregulation, mild perioperative hypothermia and postanesthetic shivering. Br Journal Anaesth, v. 84: p. 615-628, 2000.

CAMERON, M. H. Agentes Físicos na Reabilitação, da pesquisa a prática. $3^{\text {a }}$ Ed Rio de Janeiro: Elsevier, 2009.

DE ASSUnÇÃO, M. L. A. C; RAMOS, A. A. T; DE LIMA, B. A. Termoterapia profunda como tratamento fisioterapêutico na osteoartrite. Revista de Ciências Médicas, v. 19, n. 1/6, 2012. 
FERREIRA, M.G.; et al . Acurácia da circunferência da cintura e da relação cintura/quadril como preditores de dislipidemias em estudo transversal de doadores de sangue de Cuiabá, Mato Grosso, Brasil. Cad. Saúde Pública, v. 22, n. 2: p. 307-14, 2006.

GUYTON, A. C.; HALL, J. E. Tratado de Fisiologia Médica. 11. ed. Rio de Janeiro: Guanabara Koogan, 2011.

HILL, J. et al. Pulsed short-wave diathermy effects on human fibroblast proliferation. Archives of Physical Medicine Rehabiitation, v. 82: p. 832-36, 2002.

KITCHEN, S.; BAZIN, S. Eletroterapia: Prática baseada em evidências. 11ª ed. São Paulo: Editora Manole, 2003.

LONGO, G. J.; FIURINI, Jr. N. Ondas Curtas. Ed. Única, KLD Biosistemas Equipamentos Eletrônicos LTDA, 2000.

LOW, J.; REED, A. Eletroterapia Explicada: Princípios e Prática. São Paulo: Editora Manole, 2001.

MOURA, D. et al. Uso da termografia infravermelha na análise da termorregulação de cavalo em treinamento. Eng. Agricola, v. 31, n. 1: p. 23-32, 2011.

MURRAY, C. C.; KITCHEN, S. Effect of pulse repetition rate on the perception of thermal sensation with pulsed shortwave diathermy. Physiotherapy Research International, v. 5: p. 73-84, 2000.

OLIVEIRA JUNIOR, H. F. Uso da termografia na inspeção preditiva. Revista de divulgação do Projeto Universidade Petrobras e IF Fluminense, v. 1: p. 169-174, 2010. Disponível em: Disponível em: $<$ http://www.essentiaeditora.iff.edu.br/index.php/BolsistaDeValor/article/view/1811/989>. Acesso em: Mar. 2013.

OOSTERVELD, F. G.; RASKER, J. J.; JACOBS, J. W.; OVERMARS, H. J. The effect of local heat and cold therapy on the intraarticular and skin surface temperature of the knee. Seminars in Arthritis and Rheumatism, v. 35, n. 2: p. 146-51, 1992. 
PRENTICE, W. E. Modalidades Terapêuticas em Medicina Esportiva. 4. ed. São Paulo: Editora Manole, 2002.

ROBERTSON, V.; WARD, A.; LOW, J.; REED, A. Eletroterapia Explicada: Princípios e Práticas. 4. ed. Rio de Janeiro: Editora Elsevier, 2009.

SHIELDS, N. et al. Short-wave diathermy: current clinical and safety practices. Physiotherapy Research International, v. 7, n. 4: p. 191-202, 2002.

SILVA, J.; SOUZA, F. C. V.; LADEIRA, D. R.; BORGES, F. S. Análise da presença da gaiola de Faraday nos estabelecimentos de fisioterapia na cidade de Niterói - RJ. Revista Fisioterapia Ser, v. 2, n. 3: p. 10, 2007.

TEXEIRA, S. R; MESSIAS, I. P. A. F. V., MASSELlI, M. R.; CONVERSO, M. E. R. Campos Eletromagnéticos Produzidos por Equipamentos de Ondas Curtas usados em Fisioterapia: Uma Avaliação em Presidente Prudente, SP. Revista Brasileira de Fisioterapia, v. 5, n. 1: p. 35-40, 2001.

WILMORE, J. H.; COSTILL, D. L.; Kenney, W. L. Fisiologia do esporte e do exercício. Barueri: Manole, 2010. 